\title{
A leftward perceptual asymmetry when judging the attractiveness of visual patterns
}

\author{
Paul Rodway*, Astrid Schepman, Becky Crossley, \& Jennifer Lee
}

\author{
Department of Psychology, University of Chester \\ Department of Psychology \\ University of Chester \\ Parkgate Road \\ CHESTER \\ CH1 4BJ \\ United Kingdom
}

Telephone: +44 (0) 1244513479

Fax (University): +44 (0) 1244511323

\begin{abstract}
*Corresponding author
p.rodway@chester.ac.uk

a.schepman@chester.ac.uk
\end{abstract}

1416855@chester.ac.uk

1010842@chester.ac.uk

Word Count: 10756 (including reference section). 


\begin{abstract}
Perceptual judgements concerning the magnitude of a stimulus feature are typically influenced more by the left side of the stimulus than by the right side. This research examined whether the leftward bias also applies to judgements of the attractiveness of abstract visual patterns. Across four experiments participants chose between two versions of a stimulus which either had an attractive left side or an attractive right side. Experiments 1 and 2 presented artworks and experiments 3 and 4 presented wallpaper designs. In each experiment participants showed a significant bias to choose the stimulus with an attractive left side more than the stimulus with an attractive right side. The leftward bias emerged at age 10/11, was not caused by a systematic asymmetry in the perception of colourfulness or complexity, and was stronger when the difference in attractiveness between the left and right sides was larger. The results are relevant to the aesthetics of product and packaging design and show that leftward biases extend to the perceptual judgement of everyday items. Possible causes of the leftward bias for attractiveness judgements are discussed and it is suggested that the size of the bias may not be a measure of the degree of hemispheric specialisation.
\end{abstract}

Keywords: pseudoneglect, aesthetics, asymmetry, activation model, chimeric. 


\section{Experiment 1}

\section{Introduction}

When people compare two perceptually equivalent stimuli on the magnitude of a particular feature, such as darkness, they often show a bias to use the left side of a stimulus more than the right side to inform their judgement. This leftward bias is well established and has been obtained for the perceptual judgment of a range of stimulus features, including the size, brightness, and numerosity of stimuli (Charles, Sahraie, \& McGeorge, 2007; Nicholls, Bradshaw, \& Mattingley, 1999; Nicholls, \& Roberts, 2002).

Experiments that have shown leftward perceptual asymmetries in object judgement typically present participants with two equivalent versions of a stimulus, such as two luminance gradients (greyscales), with the relevant choice feature (e.g. brightness or darkness) more prominent on the left of one stimulus and on the right of the other stimulus. Participants demonstrate the bias by showing an increased tendency to select the stimulus with the choice feature that is more prominent on the left. Similar leftward biases have also been obtained in other tasks, such as letter cancellation and line bisection, and is often called pseudoneglect (Bowers \& Heilman,1980; Bradshaw et al., 1987; Charles et al., 2007; Mattingley, Bradshaw, Nettleton, \& Bradshaw 1994; Nicholls et al., 2017). It is possible that the same attentional process underlies the bias to the left side of space in pseudoneglect and to the left of objects in perceptual judgments (Mattingley et al., 1994)

While many studies have examined the leftward bias for judgements of the relative magnitude one stimulus feature (e.g. Charles et al., 2007; Nicholls, \& Roberts, 2002) other studies have shown leftward biases for judgements that require the evaluation and integration of several visual features to reach a decision. For example, leftward biases have been found 
when judging the age and attractiveness of faces (Burt \& Perrett, 1997) and have consistently been found when evaluating the intensity of facial emotional expressions (Bourne, 2010; Innes, Burt, Birch, \& Hausmann, 2015; Luh, Rueckert, \& Levy, 1991; Worley \& Boles, 2016). These findings, however, have been limited to faces and the aim of the current research was to examine whether the leftward bias for perceptual judgements that require the integration of several features generalises beyond faces to other real-world stimuli. To do this the experiments examined whether leftward biases existed for attractiveness judgements of abstract visual patterns. It was expected that the findings would facilitate an understanding of the cause and extent of perceptual asymmetries, in addition to having potential applications for the design of visual displays. The first two experiments were largely exploratory and were conducted to establish that an asymmetry in attractiveness perception existed and to measure its development in children, and the last two experiments extended the findings and eliminated alternative explanations and potential confounds.

Nicholls et al. (1999) have explained perceptual asymmetries in terms of the activation model of attentional symmetry. This proposes that activation of a hemisphere, in response to completing a function for which it is more specialised, causes an attentional bias to the contralateral side of visual space (see Kinsbourne, 1970; Kinsbourne, 1993; Luh et al., 1991; Mattingley et al., 1994; Nicholls \& Roberts, 2002; Nicholls et al., 2017; ReuterLorenz, Kinsbourne, \& Moscovitch, 1990; Spencer \& Banich, 2005). If a task activates the right hemisphere $(\mathrm{RH})$ more than the left hemisphere $(\mathrm{LH})$, there will be an increase in attention to the left hemifield, causing the left side of an item, or left side of space, to carry greater importance in the perceptual judgement. By presuming that the $\mathrm{RH}$ has a greater role in perceptual processing the activation model is able to account for why leftward biases have been found for a variety of perceptual decisions (Nicholls et al., 1999; Worley \& Boles, 2016), whilst predicting that the size of the bias for different tasks may vary because of 
varying levels of RH specialisation for each perceptual process (Nicholls et al., 2017). For example, Nicholls et al. (1999) and Luh et al. (1991) found leftward biases across a range of stimuli but relatively low correlations in the size of the biases for the same participants, which led them to conclude that distinct RH processes caused the bias for different stimuli (see also Mattingley et al., 1994).

Other explanations of the leftward asymmetry have been suggested, including a general attentional bias to the left side of space and a left-to-right scanning habit acquired from reading experience (see Bryden \& Mondor, 1991 for a discussion; Manning, Halligan, \& Marshall, 1990). For example, if participants start scanning from the left and sometimes make their choice before reaching the right side, the left of a stimulus will influence the choice more strongly than the right (Nicholls et al., 1999). The scanning explanation has been investigated by controlling scanning direction via instructions (Brodie \& Pettigrew, 1996) or visual markers (Chokron, Bartolomeo, Perenin, Helft, \& Imbert, 1998), and by measuring leftward biases in readers who habitually scan from right-to-left or left-to-right (Heath, Rouhana, \& Abi Ghanem, 2005; Vaid \& Singh, 1989; Nicholls \& Roberts, 2002). Research has shown that the direction of scanning influences the strength of the leftward bias (Chokron et al., 1998; Heath et al. 2005; Jewell \& McCourt, 2000; Vaid \& Singh, 1989) but that it exists even in illiterate participants (Heath et al., 2005) and in participants who habitually scan right-to-left if experimental controls are implemented (Nicholls \& Roberts, 2002).

Of particular relevance to the current research is the study by Chahboun, Flumini, González, McManus and Santiago (2017) which investigating the effect of reading direction and picture directionality on the aesthetic appreciation of photographs. They found that when reading direction was congruent with the directional arrangement of a photograph it increased aesthetic appreciation, which they suggest might be because greater congruence enhances 
Running Head: Leftward bias and judging attractiveness

perceptual fluency (Reber, Schwarz, \& Winkielman, 2004) and thereby aesthetic appreciation. The evidence therefore suggests that pre-existing differences in scanning direction influence the strength of leftward perceptual asymmetries and can also increase aesthetic appreciation when the directionality of a picture is congruent with scanning direction (Chaboun et al., 2017; Chokron et al., 1998; see also Chokron \& De Agostini, 2000).

The other leftward bias that has been explored extensively is for facial emotion perception using the chimeric emotional faces task (Bourne, 2010; Christman \& Hackworth, 1993; Workman, Chilvers, Yeomans, \& Taylor, 2006; Workman, Peters, \& Taylor, 2000). Chimeric emotional faces consist of a side with an emotional expression and a side with a neutral expression and it is reliably found that when a chimeric face expresses an emotion in the left-half face (from the viewer's perspective) people choose it as expressing an emotion more intensely compared to when the emotion is expressed in the right-half face. The increased selection of the left-half face has been interpreted as evidence for the RH's greater role in facial emotion processing (Bourne, 2010; Coolican, Eskes, McMullen, \& Lecky, 2008; Levy, Heller, Banich, \& Burton, 1983; Watling \& Bourne, 2013; Workman et al., 2000). While there are other possible causes of perceptual asymmetries in the chimeric faces task (Bryden \& Mondor, 1991) including a general RH superiority in configural perception (Burt \& Perrett, 1997), evidence suggests it is due to the RH's specialised role in emotion perception, but that a general attentional bias to the left side of space can contribute to the size of the effect (Innes et al., 2015; see also Luh et al., 1991).

For the current research two equivalent versions of a stimulus were constructed with one version having an attractive left side and the other having an attractive right side. The first two experiments used chimeric abstract artworks and the third and fourth experiments used wallpaper designs. Each chimeric stimulus was created by joining together an attractive 
abstract artwork and an unattractive abstract artwork to form a single artwork. It can be noted that examples of chimeric art have been produced by some artists, most notably by David Salle, so the idea of having two different sides to an artwork is not entirely unusual.

The attentional activation model predicts that an asymmetry in the involvement of the brain's hemispheres in the perceptual evaluation of visual patterns may bias choice to one side of an item when judging its attractiveness. Evidence for a greater role of the RH in aesthetic judgements is equivocal (Zaidel, 2013, 2015), but a substantial body of research suggests that the RH may have a greater role than the $\mathrm{LH}$ in a number perceptual processes that may be central to perceiving attractiveness (Kimura, 1961; Bryden, 1982; Oltedal \& Hugdahl, 2016), such as processing symmetry (Wright, Makin, \& Bertamini, 2017) and the global configuration of visual elements (Boles \& Karner, 1996; Bradshaw \& Nettleton, 1981; Nebes, 1978; Van Kleeck, 1989). Based on this research and other studies demonstrating leftward biases in a variety of perceptual judgements (e.g. Chaboun et al., 2017; Luh et al., 1991; Worley \& Boles, 2016) we expected a leftward asymmetry would also be present for the judgement of the attractiveness of abstract visual patterns. We therefore predicted that an artwork with the attractive side on the left would be preferred more than an artwork with the attractive side on the right. Conversely if the position of the attractive/unattractive side does not influence choice it would show that attractiveness judgements of visual patterns did not show a leftward bias.

\section{Method}

\section{Participants}


Thirty-four participants ( 31 females, 3 males) from the University of Chester took part in the experiment. Individuals were recruited by opportunity sampling or by use of the department's "Participant Pool" (in the latter case, participation credit was awarded). Age ranged from 18 to 56 (mean age $=21, \mathrm{SD}=6.4$ ) and 32 were right-handed and 3 were lefthanded (by self-report). Ethical approval for this experiment, and all subsequent experiments, was provided by the University of Chester which complied with British Psychological Society ethical guidelines.

\section{Stimuli and Chimeric art task}

Thirteen abstract artworks were selected from a set of artworks (see Schepman, Rodway, Pullen, \& Kirkham, 2015) that had been pre-rated for attractiveness using a 7 point Likert scale (with 1 being 'unattractive' and 7 being 'attractive'). Seven of the artworks were selected to have low attractiveness (mean attractiveness rating $=2.55$, range $2.28-2.89$ ) and six to have high attractiveness (mean $=4.35$, range $3.89-4.74$ ). They were selected because they represented the most attractive and least attractive artworks in the set.

Each attractive artwork was combined with each of the unattractive artworks to create 42 chimeric artworks. The side of each chimeric artwork that was attractive/unattractive was then transposed, so that the attractive/unattractive artwork was on the left side and the right side equally often, creating two versions of each chimeric artwork, and 84 in total. In most studies that have examined leftward biases mirror reversal has been used to create the second chimeric stimulus, especially for the chimeric facial emotion task where mirror reversal is a necessity because it ensures that the two equivalent stimuli are still identifiable faces. Other studies, however, have occasionally not used mirror reversal and have still obtained leftward biases (e.g. Luh et al.,'s 1991 dot number task used 180 degree rotation and Nicholson et al., 
1999 used equivalent stimuli rather than mirror reversed greyscales). In this experiment it was not necessary to use mirror reversal and it would have had the negative consequence of reversing the overall configuration of an artwork's pattern in the second (reversed) stimulus, potentially influencing its attractiveness. We therefore decided to keep each side of the chimeric artwork identical in both versions, to ensure that the sides and their attractiveness were equivalent and matched their presentation when originally rated for attractiveness. However, a negative consequence of this approach is that the join of the two sides at the midline differed between the two versions. Despite this, we felt that this approach was better than mirror reversal as it was unlikely that a difference at the midline would systematically cause a bias to one side.

The standard forced choice chimeric task was used so that the two versions of the same chimeric artwork were presented on each trial, one above the other (See Figure 1, we are unable to reproduce the stimuli for copyright reasons but they are available in the supplementary materials). To ensure that participants maintained their attention during the task, and the task was not too taxing for the child participants in experiment 2 (which was run concurrently with experiment 1 ), the 84 trials were separated into two blocks of 42 trials. The position (top or bottom) of the chimeric artworks was counterbalanced with participants presented with both versions within a block of trials. Half of the participants completed one block and half completed the other block and for each block the trials were presented in a random order.

The chimeric artworks were presented on a computer using E-Prime software. They were presented on screen in colour on a white background. The dimensions of the original artworks that were combined to form the chimeric artworks were adjusted to enable the sides of each chimeric artwork to be the same size. Each chimeric artwork was $12.4 \mathrm{~cm}$ wide $(6.2$ 
$\mathrm{cm}$ per side) and $5.3 \mathrm{~cm}$ high, with the two chimeric artworks separated by $2.4 \mathrm{~cm}$. The artworks were presented centrally and participants sat approximately $50 \mathrm{~cm}$ from the screen.

---Insert Figure 1 about here ---

\section{Procedure}

It was explained to the participants that they would be presented with novel artworks, made by joining two previously separate abstract artworks, and that we were only interested in their preferences. The following instructions were presented at the start of the trials: "In the centre of the screen there will be $a^{\prime}+{ }^{\prime}$ which we would like you to look at. You will then be presented with two abstract artworks, one above the other. We would like you to choose the artwork that you find most attractive. Please press ' $k$ ' if you find the top artwork most attractive. Please press ' $m$ ' if you find the bottom artwork most attractive. All of the artworks are novel and they are a combination of two artworks that were created independently by different artists. We are interested in which novel artworks are liked by participants. There are no right or wrong answers, we are only interested in what you find attractive. You will be presented with 42 artworks in total."

Each participant was randomly allocated to one block of trials and was tested individually. On each trial a fixation cross (font 32) was presented in the centre of the screen for 1 second. This was then replaced by two opposite versions of the same chimeric artwork, one above the other. The stimuli were removed when the participant responded and then replaced by a pattern mask for 500 milliseconds, followed by the fixation cross, which signalled the start of the next trial. 


\section{Results}

The standard way of calculating laterality quotients was adopted (see Bourne 2008, 2010) by applying the following equation: (Number of left side choices when the attractive side was on the left - (Total number of trials - Number of left side choices when the attractive side was on the left)/ Total number of trials). This calculation produced scores ranging from -1 (always choosing the chimeric artwork with the attractive right side) to +1 (always choosing the chimeric artwork with the attractive left side).

The mean laterality quotient was $+.175,(\mathrm{SE}=.075)$, with participants showing a bias to choose the chimeric artwork with an attractive left side more often than the chimeric artwork with an attractive right side. A one sample t-test showed that the laterality quotient was significantly different from zero (where zero would indicate no bias), $t(33),=2.33, p<$ $.013 ; d=.40$, clearly showing a leftward bias for attractiveness judgements of abstract artworks. To ensure that the results were not due to the presence of three left handers in the sample we re-ran the analysis with these participants excluded and again obtained a significant leftward bias, $t(30),=1.82, \mathrm{p}=.039 ; d=.33$ (one tailed).

We checked that each block of trials showed a similar laterality quotient. Unexpectedly the first block showed a significant leftward bias, $t(16),=3.08, \mathrm{p}=.004 ; d=$ .76 (one tailed), whereas the second block did not, $t(16),=.3, \mathrm{p}=.38$. This demonstrated that the leftward bias was carried primarily by the first block of trials. To examine the reason for this we calculated the leftward bias for each of the 84 arrangements of stimuli, and found that 59 arrangements showed a leftward bias whereas 25 elicited a rightward bias, and by chance those that those elicited a rightward bias were more common in the second block of trials (19 in block two and 6 in block one). An examination of the chimeric stimuli indicated that the 
leftward bias was attenuated when the difference in perceived attractiveness between the left and right sides was reduced. We tested this by correlating the mean leftward bias elicited by each chimeric stimulus (calculated by taking the average leftward bias for the original and transposed version, with negative scores reflecting a rightward bias), with the difference in perceived attractiveness between the right and left sides of each chimeric stimulus (calculated by taking the difference score of the attractiveness ratings of each side of the stimulus). This revealed a significant positive correlation. $\mathrm{r}=.34, \mathrm{~N}=42, \mathrm{p}=.027$, two tailed, showing that a larger leftward bias was associated with a larger difference in perceived attractiveness between the two sides.

\section{Discussion}

When participants chose between two abstract chimeric artworks they were more likely to select the artwork with the attractive side on the left rather than the right. These results demonstrate, for the first time, that the leftward bias occurs for the judgement of the attractiveness of abstract artworks.

On average the participants chose the artwork with an attractive left side $59 \%$ of the time which is smaller than the average leftward bias of $68 \%$ obtained by Nicholls et al. (1999) across a variety of stimuli. The observed laterality quotient of .175 is similar to that found by Bourne (2010) for the emotion sadness (.16) and to that by Innes et al. (2015) for the six basic emotions combined (.17), but it is not as large as the bias typically obtained for some basic emotions, such as happiness (.41, Bourne, 2010), fear (.45, Bourne, 2010) and sadness (.30, Innes et al., 2015), or for the chimeric dot task (.29, Luh et al., 1991; see Voyer, Voyer, \& Tramonte, 2012). 
One reason why the leftward bias may have been smaller than for other tasks is because the difference between the 'attractive' and 'unattractive' sides was more subjective than for greyscales and chimeric emotional stimuli, where each individual stimulus has an objectively darker or more emotional side than the other. As art appreciation has a degree of subjectivity (McManus, 1980) on some trials participants will have preferred the 'unattractive' side to the 'attractive' side, which will have increased variability in the data and weakened the leftward bias for attractiveness judgements. This interpretation is supported by the finding that the size of the leftward bias correlated with the level of difference in attractiveness between the sides. The implications of this finding are explored more fully in the general discussion. Finally, it is also possible that if the cause of the leftward bias is due to differences in hemispheric specialisation, then perhaps the RH is only slightly more specialised than the LH for judging the attractiveness of abstract artworks.

\section{Experiment 2}

\section{Introduction}

The second experiment examined the developmental time course of the leftward bias in attractiveness judgements. Previous research that has measured the development of leftward biases has often used the chimeric emotion task and has found that the bias develops at different ages for different emotions. For example, a leftward bias for the perception of happy expressions has been detected at age 5 to 6 (Levine \& Levy, 1986), but for other basic emotions leftward biases appear to develop at ages 7 to 8 , becoming fully developed and equivalent to adults at ages 10 to 11 (Taylor, Workman, \& Yeomans, 2012; Watling \& Bourne, 2013; Workman et al., 2006). One interpretation of these findings is that the RH 
becomes specialised for the perception of different emotions at different ages and to different extents (see Bourne, 2010; Watling \& Bourne, 2013; Workman et al., 2006).

Other lateralised processes also take time to develop and show wide variation in the age that they reach adult levels of lateralization (Chiang, Ballantyne, \& Trauner, 2000). Boles, Barth, and Merrill (2008) propose that brain maturation, particularly of the corpus callosum, and experiential factors determine when a function becomes lateralised. They suggest that both of these factors underpin individual differences in the development of lateralisation. It appears that the majority of lateralised processes reach adult levels around the age of 12, with most processes lateralised earlier than this (see Boles et al., 2008 for a review). The leftward bias in experiment 1 was equivalent in size to emotions that tend to become lateralised at later ages (e.g. disgust), and was smaller than for emotions which become lateralised earlier (e.g. happiness). If the leftward bias in attractiveness judgements follows a similar development time course to the leftward bias in emotion perception and other lateralised processes, then we can expect it to become stronger with age, so that lateralisation may not be present at 6 or 7 , but becomes evident at older ages, approaching adult levels at age 10-11. The second experiment explored this prediction and also served as a partial replication of the first experiment.

\section{Method}

\section{Participants}

The participants were 87 children from a primary school in the Greater Manchester region in an area of low socio-economic status (measured by the school's Income Deprivation Affecting Children Index). They comprised 27 children aged 6-7 (15 males, 12 females), 30 children age 9-10 (21 males, 9 females), and 30 children aged 10-11 (16 males, 
14 females). The school head teacher gave consent for the study and children were then recruited from classrooms with parental opt-out consent.

\section{Procedure}

The stimuli and task were the same as in Experiment 1 and the experiment was run on a laptop computer. Participants were tested individually in a familiar quiet room by a teacher who they knew (author BC), with each participant completing 42 trials. Before starting the experiment, participants were told that we were interested in which pictures they find attractive and they were presented with an example of the stimuli they would see on the computer screen. The instructions were simplified from the first experiment and consisted of the following "You will be shown two pictures, if you like the top picture most press the ' $k$ ' key. If you like the bottom picture most press the 'm' key".

\section{Results}

The laterality quotient for each participant was calculated using the equation described in experiment 1 and the mean laterality quotients for each age group are presented in Table 1. To test the hypothesis that the development of the leftward bias increased across the age groups a linear trend analysis was run. The weighted option was selected due to a slight inequality in group sizes. This showed a significant increase in laterality quotient with age, $F(1,84)=4.57, \mathrm{p}=.035$, and non-significant deviation, $F(1,84)=.006, \mathrm{p}=.939$. To put the analysis on an equal footing with that for experiment 1, one sample t-tests were also run for each group on an a priori basis. These showed that the leftward bias was significantly different from zero for the children aged 10 to $11, t(29)=2.5, \mathrm{p}<.018, d=.46$, but not for 
the children aged 9 to 10 , or aged 6 to 7 . Therefore, a significant leftward bias for the liking of abstract artworks emerged at age 10 to 11 .

--- Insert Table 1 here ---

\section{Discussion}

There was evidence that the leftward bias in attractiveness judgements takes time to develop, being present in children aged 10-11, but not younger children. The lack of bias in the younger age groups shows that it does not occur automatically in all circumstances and suggests that it is due to a developmental change. The bias in 10-11 year olds was also smaller than obtained in adults for the first experiment (.12 vs .17) perhaps indicating that it reaches adult levels at a slightly older age.

It is noticeable that the age of onset of the leftward bias is similar to that obtained for some basic emotions (Watling \& Bourne, 2013; Workman et al., 2006) and other functions which do not become fully lateralised until age 11 (Boles et al., 2008). An interesting question for future research is whether a leftward bias for attractiveness judgements of other stimuli, such as faces, has a similar developmental onset, perhaps pointing to this age as a key time for the development of perceptual asymmetries in attractiveness processing. The reason why the asymmetry develops at this age is likely to be due to both brain maturation and greater experience with relevant tasks (Boles et al., 2008), including producing and 
evaluating art and with reading which promotes stronger habitual left-to-right scanning (De Agostini et al., 2010).

\section{Experiment 3}

\section{Introduction}

This experiment had three aims. First to replicate the leftward bias in attractiveness judgements with a different and more naturalistic task. Second to examine whether it was caused by asymmetries in the perception of the colourfulness of the stimuli, and third to eliminate the possibility that the leftward bias was caused by an asymmetry in hand response.

The attractiveness of abstract art depends on the configuration of a number of attributes, including colourfulness, symmetry, and complexity, as well as the individual preferences of the perceiver (Leder, Belke, Oeberst, \& Augustin, 2004; Pelowski, Markey, Lauring, \& Leder, 2016; Schepman et al., 2015). It can be suggested that the leftward bias was not due to attractiveness judgements per se but to participants perceiving a particular attribute of the artwork more strongly when on the left. For example, in experiments 1 and 2 the attractive side of each stimulus tended to be more colourful than the unattractive side because colourfulness is a typical property of attractive abstract art. As there is some evidence for the RH having a greater role in colour processing (Sasaki, Morimoto, Nishio, \& Matsuura, 2007; but see Danilova \& Mollon, 2009), the leftward bias might therefore have been due to the greater impact (or salience) of the colour of the stimulus when on the left side than the right side.

While we do not feel that this interpretation negates the basic finding of a leftward bias in attractiveness judgements it was important to examine the possibility that the bias reflected an asymmetry in the perception of colourfulness rather than attractiveness per se. It 
can be noted, however, that perceptual attributes are not fully independent of attractiveness, with stimulus complexity, for example, sometimes viewed as directly related to levels of aesthetic appreciation (Berlyne, 1963; Roberts, 2007).

In this experiment wallpaper designs, pre-rated for attractiveness, were used as stimuli rather than abstract artworks. On each trial two equivalent arrangements of wallpaper designs were presented to participants, with one arrangement having an attractive/unattractive design on the left/right and the other arrangement having these same designs but on the opposite sides. To test the possibility that the leftward bias was due to an asymmetry in perceiving the colourfulness of the stimuli, two sets of stimuli were formed, with the first colour set consisting of items with one or more colours (other than black and white), and the second non-colour set consisting only of black and white or greyscale items. If the leftward bias was due to perceiving the presence of colour more intensely on the left than on the right then the bias should only be present for stimuli with colour.

A further issue that could have confounded the results of experiments 1 and 2 is that participants used their dominant hand to make a response (the right hand for most subjects) which might have caused an attentional asymmetry (see Nicholls \& Roberts, 2002 for a discussion). To discount this possibility participant were asked to make a bimanual response when they selected the arrangement they found most attractive.

One advantage of using wallpaper designs was that many of them were repeating patterns so that the join at the midline tended to be very similar or identical in the original and transposed version of each arrangement. This reduced the likelihood that the leftward bias was due to a difference in the midline between stimuli. In addition, the wallpaper designs also enabled the examination of the generalisability of the leftward bias in a more ecologically valid test, because judging the attractiveness of two adjacent designs is something that people 
do in the real world. We predicted that a leftward bias would be obtained for attractiveness judgements of wallpaper arrangements and that it would be unaffected by the presence of colour.

\section{Participants}

Twenty-nine participants (26 females, 3 males) from the University of Chester took part in the experiment. Individuals were recruited by opportunity sampling or by use of the department's "Participant Pool" (in the latter case, participation credit was awarded). Age ranged from 18 to $46(\mathrm{M}=23.7 \mathrm{SD}=7.05)$ and 27 were right-handed and 2 were left-handed (by self-report).

\section{Stimuli}

A total of 54 images of wallpaper designs selected from variety of online images using Google search. Some designs had one or more colours, others were in black and white or greyscale, and some designs that were originally in colour were changed to greyscale. The designs were then printed in two booklets, with the first consisting of 25 designs and the second 29 designs, with each design presented centrally on a page. The attractiveness of each design in each booklet was rated by 9 participants (different participants for each booklet) using a 7 point Likert scale (with the anchors being Unattractive and Attractive). From these ratings 8 of the most attractive designs were selected (mean attractiveness rating 4.33, range $=4.1-4.7$ ), four of which contained one or more colours (other than black and white) (mean rating $=4.33$, range $=4.2-4.4$ ), and four in black and white or greyscale (mean rating $=$ 4.33, range $4.11-4.7$ ). Eight of the most unattractive designs were also selected (mean rating $=1.8$, range $=1.4-2.3$ ), four of which contained one or more colours (mean rating $=$ 
Running Head: Leftward bias and judging attractiveness

1.8 , range $1.4-2.5$ ) and four in black and white or greyscale (mean rating $=1.83$, range $=$ $1.56-2.3)$.

The four attractive colour designs were then combined with the four unattractive colour designs to create 16 wallpaper arrangements. The side of each arrangement that was attractive/unattractive was then transposed, so that the attractive/unattractive design was on the left and the right side equally often, creating two versions of each wallpaper arrangement and 32 in total. The same combinations were created for the four attractive and four unattractive non-colour designs, creating a further 32 wallpaper arrangements in total. The standard forced choice chimeric task was used so that the two equivalent versions of the same wallpaper arrangement were presented on each trial, one above the other (see Figure 1). The position (top or bottom) of the wallpaper arrangements occurred equally often within the set of trials and each participant completed 64 trials in total $(32$ with colour designs and 32 with non-colour designs). The dimensions of the wallpaper arrangements, including the size of each side and their separation were the same as the stimuli presented in experiments 1 and 2, as was the participant's seating distance from the monitor.

\section{Procedure}

The experiment was run on a laptop computer with E-Prime software and the participants were tested individually. Each participant was presented with the following instructions at the start of the trials: "In the centre of the screen there will be $a$ ' + ' which we would like you to look at. You will then be shown two wallpaper designs side-by-side, in the same way that they might appear next to each other on different walls in a room. The wallpaper designs will be arranged so that each design is either on the left or right. We would like to know which of the two arrangements you find most attractive. If you find the top 
Running Head: Leftward bias and judging attractiveness

arrangement most attractive please press the ' $k$ ' and ' $d$ ' keys at the same time. If you find the bottom arrangement most attractive please press the ' $m$ ' and ' $c$ ' keys at the same time." The timing of the presentation of the stimuli was the same as for experiments 1 and 2 and the 64 trials were presented in a random order.

\section{Results}

The mean laterality quotient was calculated which gave a score of $+.42,(\mathrm{SE}=.078)$, with participants showing a bias to choose the arrangement with an attractive design on the left side more often than the arrangement with an attractive design on the right side. A one sample t-test showed that the laterality quotient was significantly different from zero (where zero would indicate no bias), $t(28),=5.42, \mathrm{p}<.0001 ; d=1$, showing a clear leftward bias when judging the attractiveness of wallpaper designs. The bias was also present when the data from the two left-handed participants was removed, $t(26)=4.9, \mathrm{p}<.0001 ; d=.95$.

The mean laterality quotient for the colour arrangements $($ Mean $=.409, \mathrm{SE}=.087)$ was similar to the laterality quotient for the non-colour arrangements $($ Mean $=.422, \mathrm{SE}=$ .081) and a paired samples t-test showed no significant difference in laterality quotients for these sets of stimuli, $t(29)=.18 . \mathrm{p}=.86$. The participants' laterality quotients for non-colour and colour arrangements also correlated strongly, Spearman's rho $=.714, \mathrm{~N}=29, \mathrm{p}<.001$, two tailed, showing that individual participants showed a consistent bias to prefer the arrangement with an attractive left-side for both colour and non-colour stimuli.

To further examine the influence of the attributes of the wallpaper designs on the leftward bias 14 additional participants rated each wallpaper design, on a 7 point Likert scale, for complexity (anchors: simple, complex), luminance (anchors: dull, bright), and 
colourfulness (anchors: not colourful, very colourful). The mean ratings are presented in Table 2.

---Insert Table 2 about here ---

In line with expectations, non-parametric Wilcoxon Signed Ranks tests showed significant differences in colourfulness between the attractive colour and attractive noncolour designs $(\mathrm{Z}=-2.94, \mathrm{p}=.003)$, and between the unattractive colour and unattractive non-colour designs $(\mathrm{Z}=-2.8, \mathrm{p}=.005)$. We also analysed differences between the attractive and unattractive sides of each arrangement for the attributes of complexity, luminance, and colourfulness. For the colour arrangements the difference between the attractive and unattractive sides for perceived complexity $(Z=-.85, p=.39)$, luminance $(Z=-.43, p=.66)$ and colourfulness $(\mathrm{Z}=-1.9, \mathrm{p}=.057)$ were not significant, although for colourfulness it might have become significant with more data. For the non-colour arrangements the difference between the attractive and unattractive sides for perceived luminance $(Z=-1.0, p$ $=.3$, ) and colourfulness $(\mathrm{Z}=-.51, \mathrm{p}=.61)$ were not significant, but was significant for perceived complexity $(Z=-2.1, p=.036)$, with the attractive side viewed as more complex. These results suggest that for the colour arrangements perceived differences in luminance and complexity between the sides were not responsible for causing the leftward bias. In addition, for the non-colour arrangements perceived differences in luminance and colourfulness between the sides do not appear to be responsible for the leftward bias. 


\section{Discussion}

In replication of experiments 1 and 2 a leftward bias was obtained for attractiveness judgements, showing that it generalised to another class of stimuli and a slightly different task. The results also showed that the leftward bias was not an artefact of the hand used to give the response and was not determined by the colourfulness of stimuli. The additional rating data also suggested that differences in perceived luminance between the attractive and unattractive sides did not cause the leftward bias. The possible influence of stimulus complexity is discussed more fully in the next experiment.

The leftward bias was very robust, with 24 of the 29 participants showing the bias and with participants, on average, choosing the left-attractive configuration $70 \%$ of the time. This size of leftward bias is equivalent to that reported with the greyscales task by Nicholls et al. (1999) and is larger than obtained with the abstract artworks in the first experiment. A plausible reason for this is that the mean difference in attractiveness between the two sides was larger, and therefore possibly less subjective in this experiment compared to the first (2.51 vs 1.8 ), which will have increased the consistency of the perceived difference in attractiveness between the two sides and resulted in a stronger leftward bias. In addition, the effect may have been more robust because the task was more familiar and ecologically valid, with a clear purpose and rationale, potentially reducing any tendency by the participants to 'second guess' what the study might be trying to measure.

The difference in strength of the leftward bias across experiments 1 and 3 concords with previous research which has shown similar variations in the size of the effect with different stimuli (e.g. Nicholls, et al. 1999; Luh, et al., 1991; Burt \& Perrett, 1997). Such variability suggests that perceptual asymmetries are not a simple product of scanning 
direction, because if that was the case similar sized biases might be expected for different tasks and stimuli.

\section{Experiment 4}

\section{Introduction}

Experiment 3 showed the leftward bias did not depend on the colourfulness of the designs but other attributes that contribute to attractiveness, such as complexity, were not controlled for directly. Although the additional rating data suggested that for the colour arrangements the leftward bias was not due to differences in perceived complexity or luminance, for the non-colour stimuli there was a difference in perceived complexity between the attractive and unattractive sides, and this could have caused the leftward bias for these arrangements.

To examine whether an asymmetry in complexity perception underpinned the leftward bias we decided to re-run the experiment with the attractive and unattractive sides of each arrangement matched for complexity. However, it became apparent when selecting the stimuli, that because colourfulness and complexity both contribute to attractiveness it was difficult to match attractive designs with unattractive designs for both of these attributes at the same time, because a reduction in both attributes reduced attractiveness. Moreover, for the non-colour stimuli, where colourfulness did not contribute to attractiveness, the complexity of the design was related to attractiveness, making it difficult to match the attractive and unattractive designs for complexity. For these reasons it was decided to only use colour designs, matched for complexity, but not matched for colourfulness. Based on the view that the leftward bias was caused by an attractiveness judgement, rather than an 
asymmetry in complexity perception, it was expected that the bias would still be present when the attractive and unattractive sides were matched for complexity.

\section{Method}

\section{Participants}

Forty-five participants (29 female, 16 male), from the University of Chester took part in the experiment. Individuals were recruited by opportunity sampling or by use of the department's "Participant Pool" (in the latter case, participation credit was awarded). Age ranged from 19 to $58(\mathrm{M}=30.5, \mathrm{SD}=10.6)$ and 39 were right-handed and 6 were left-handed (by self-report).

\section{Stimuli}

Six colour wallpaper designs were selected, three of which were attractive and three unattractive, which were matched, as closely as was possible, for levels of perceived complexity (see Table 3 for the ratings of each design).

---Insert Table 3 about here ---

Each attractive design was paired with an unattractive design to create 9 wallpaper arrangements. The side of each arrangement that was attractive/unattractive was then transposed, so that the attractive/unattractive design was on the left and the right side equally often, creating two versions of each wallpaper arrangement, and 18 in total. The standard 
forced choice chimeric task was used so that the two versions of the same wallpaper arrangements were presented, one above the other (see Figure 1). The position (top or bottom) of the wallpaper arrangements was also counterbalanced with participants presented with both versions within a session. The 18 arrangements were printed in a booklet and three versions of the booklet were produced so that the order of the wallpaper arrangements varied across participants. A response sheet was prepared which consisted of a response box next to a small image of each wallpaper arrangement.

\section{Procedure}

Each participant was tested individually. It was explained to them that on each page of the booklet they would be presented with two arrangements of wallpaper designs, as they might appear on the walls of a room, and to choose the arrangement that they found most attractive. The participants registered their preference by ticking the appropriate box on the response sheet. Each participant completed one booklet and the allocation of the booklets was counterbalanced across participants.

\section{Results}

The participants' responses were collated and the mean laterality quotient was calculated giving $.14(\mathrm{SE}=.053)$, with participants showing a bias to choose the arrangement with an attractive design on the left more often than the arrangement with an attractive design on the right. A one sample t-test showed that the laterality quotient was significantly different from zero $t(44),=2.68, \mathrm{p}=.005 ; d=.40$, one tailed, showing a leftward bias in attractiveness judgements. Unfortunately, the participant's handedness information became separated from their response sheet so we were unable to run the analysis with the left 
handers excluded. However, based on the results of experiments 1 and 3 it would be reasonable to assume that the results would have been unaffected.

\section{Discussion}

Once again participants showed a bias to choose the wallpaper arrangement with an attractive left side more often than an attractive right side. As the attractive and unattractive designs were matched for complexity the results suggest that the leftward bias in attractiveness judgements were not caused by an underlying asymmetry in complexity perception. The results also showed that the effect generalises to designs presented on paper, although it was slightly smaller than in the previous experiment, perhaps because fewer stimuli and trials were used, or because of other factors, such as the presentation conditions on paper being more variable than with a computer.

\section{General Discussion}

A leftward bias in attractiveness judgements of visual patterns was obtained across four experiments. It was present for both abstract artworks and wallpaper designs and did not appear to be caused by a systematic asymmetry in perceiving one specific attribute of a pattern, such as colourfulness, complexity, or luminance. It was also found that the bias develops around the age of $10-11$, which is a relatively late age in comparison to other asymmetries described in the literature (see Boles et al., 2008). This might be because judging the attractiveness of complex visual patterns relies on a variety of late maturing perceptual processes and experiences. 
The leftward bias is well established for perceptual judgements involving the relative magnitude of an individual perceptual feature (Nicholls \& Roberts, 2002). It is also routinely found when judging faces on dimensions of attractiveness, emotional expression, and age (Burt \& Perrett, 1997; Bourne, 2010); judgments which require the integration of multiple perceptual features. This latter result has frequently been interpreted as being due to the RH's specialisation for facial stimuli (Innes et al., 2015), from which it could be inferred that the leftward bias for complex perceptual judgments is unique to faces. However, judging the attractiveness of an abstract visual pattern also relies on the ability to evaluate multiple attributes of a pattern and how they are configured (Leder, Belke, Oeberst, \& Augustin, 2004). Therefore, the current findings show that the leftward bias is not limited to simple judgments of the relative magnitude of a stimulus feature, or to faces when complex judgments are required, but generalises to integrative perceptual judgements of stimuli which are not typically regarded as being specialised to the RH. An implication of the apparent generalisability of the leftward bias is that it might be present for the perceptual judgment of many other non-facial stimuli that rely on the evaluation of multiple features.

The attractive and unattractive sides of the wallpaper arrangements had similar levels of colourfulness in experiment 3 and were matched for complexity in experiment 4 . This indicates that neither colourfulness and complexity individually and systematically caused the perceptual asymmetry. Clearly, however, each attribute contributes to attractiveness and it is likely that when the strength of one attribute (e.g. colourfulness) was similar between the sides the importance of other attributes (e.g. complexity) for the judgment of attractiveness increased. Therefore, while each attribute does not appear to have individually caused the bias, they will have influenced the bias by contributing to the attractiveness of a design. It is, however, also important to emphasise that leftward biases seem to be produced by the judgement applied to the stimulus rather than the stimulus features themselves, with, for 
example, the same greyscale stimuli eliciting a leftward bias for both brightness and darkness judgements (Nicholls et al., 1999). Similarly, in the current study, as participants were asked to judge attractiveness it is likely they were doing this by evaluating the different attributes of a pattern that contributed to attractiveness rather than showing a consistent perceptual asymmetry to one attribute.

Several explanations of perceptual asymmetries have been proposed, including scanning direction and a general attentional bias to the left (see Bryden \& Mondor, 1991; Jewell \& McCourt, 2000; Nicholls et al., 1999) and it is possible that more than one process contributes to the effect. As noted earlier, left-to-right scanning could cause the leftward bias because it might increase the influence of the left side of a stimulus on the perceptual decision (Nicholls et al., 1999). This would provide a parsimonious explanation of the leftward bias and its emergence in older children who have more reading experience. In addition, habitual left-to-right scanning has been found to influence the aesthetic appreciation of pictures when the directionality of a picture is congruent with scanning direction (Chahboun et al., 2017; Chokron \& De Agostini, 2000; Ishii, Okubo, Nicholls, \& Imai, 2011). It is possible that the arrangement of an attractive side/unattractive side is more congruent with a left-to-right reading direction than is the reverse arrangement, which enhances perceptual fluency and aesthetic appreciation (see Chahboun et al., 2017). This process could therefore underpin the leftward bias obtained in the current research. To examine these explanations future research could measuring eye movements during the task or use participants who read from right-to-left.

A further account that has received wide ranging support is that the leftward bias is due to differences in hemispheric specialisation causing an asymmetry in attentional allocation (Nicholls et al., 1997). As described previously, a body of research suggests that the RH may have a greater role than the LH in perceptual processes that are central to judging 
attractiveness (Bradshaw \& Nettleton, 1981; Bryden, 1982; Kimura, 1961; Nebes, 1978; Oltedal \& Hugdahl, 2016; Van Kleeck, 1989; Wright et al., 2017). Luh et al. (1991) also concluded from their research that the RH is more specialised for processing some stimuli, such as facial emotion, but that it is also more specialised than the LH for visual processing in general. It is therefore possible that judging the attractiveness of a visual pattern recruits the RH more than the LH, causing an attentional bias to the left side of an item and thereby influencing the attractiveness decision.

The finding that a larger difference in the level of attractiveness between the left and right sides increased the strength of the leftward bias is important because it might also have occurred for other leftward biases reported in the literature. For example, in the chimeric emotional faces task the strongest perceptual asymmetry is often obtained with happy expressions (Bourne, 2010; Workman et al., 2006) which is the expression that is detected fastest and possesses the most salient features (Calvo, \& Nummenmaa, 2008). It is possible that the relative perceptual difference between the sides (e.g. between a happy and a neutral expression, compared to the difference between disgust and neutral) influences the size of the leftward bias for the emotion chimeric task. This may apply to studies using other stimuli (e.g. Luh et al., 1991), with the size of the perceptual asymmetry being determined by the relative difference in the strength of the judgement feature between the sides of a stimulus, rather than being due to differences in the degree of hemispheric specialisation for processing a particular feature. This is speculative and needs to be examined further but it might explain why studies have found a weak correlation in leftward biases across different stimuli.

\section{Limitations and Future Research}


The robustness of the leftward bias found in experiment 3 suggests that this may be a useful task with which to explore how different visual properties influence perceptual asymmetries. An interesting question for future research is whether the leftward bias generalises to the judgment of the attractiveness of other types of images such as representational pictures. Research suggests that the $\mathrm{LH}$ is more specialised for interpreting and assigning meaning to information (Beaumont, 1985; Gazzaniga, 1998; Marinsek, Turner, Gazzaniga, \& Miller, 2014; Zaidel \& Edelstyn, 2009) with occipito-temporal regions of the LH processing visual information semantically (Rossion et al., 2000). This may make the LH more suitable for appreciating and evaluating the attractiveness of representational pictures (see also Zaidel \& Kasher, 1989), whereas the RH's greater involvement in perceptual and visuo-spatial processing may make it more specialised for the perceptual appraisal of a pattern's elements and how they are configured to form a whole. If future research obtains a rightward bias for attractiveness judgements of representational images and a leftward bias for abstract visual patterns, it would support the view that hemispheric specialisation is causing the effect.

The presence of a leftward bias in attractiveness judgements also has clear applications, particularly in the design of product packaging, labels, and other forms of visual displays where attractiveness is important. Research has shown that people tend to look first and longer at the centre of a display, or scene (Bindemann, 2010), and to regard centrally placed items as more important and preferable (Atalay, Bodur, \& Rasolofoarison, 2012; Kreplin, Thoma, \& Rodway, 2014; Rodway, Schepman, \& Lambert, 2012). The current findings, in addition to other research, indicate that for an array of display items those in the centre with an attractive left side may be the most preferred. There are, however, many questions to explore with regard to the leftward bias in applied settings, including the factors 
that determine its presence, such as the type of visual information it applies to, and whether it influences consumer choices.

There are also aspects of this research which could be improved. There was an imbalance in the number of male and female participants in experiments 1,3 , and 4 , which was due to having more female psychology students who volunteered for the study. Therefore, we cannot be certain that the leftward bias generalises to males, although it can be noted that no other study has reported a sex difference in leftward biases, and the results of experiment 2 , where there were similar numbers of male and female participants, indicate that the effect is not specific to females.

\section{Conclusion}

The leftward bias in attractiveness judgments of visual patterns is robust and appears to be a reflection of the judgement of attractiveness, rather than a specific attribute that contributes to attractiveness. The results add to the evidence of asymmetries in perceptual processing, showing that they extend beyond faces to complex perceptual judgements of realworld stimuli. This suggests that similar asymmetries in perceptual judgements may be present for a range of other stimuli that have not previously been examined. The findings also have wide-ranging implications for the display of consumer products by showing that the attractiveness of an item is influenced more by its left side than its right side. It is suggested that the cause of the leftward bias may be habitual left-to-right scanning or greater specialisation of the right hemisphere for attractiveness judgements. Finally, while the presence of a leftward bias might reflect right hemisphere specialisation, because the relative difference in attractiveness between the sides influenced the strength of the leftward bias, we 
Running Head: Leftward bias and judging attractiveness

suggest that the size of a leftward bias probably should not be taken as a measure of the degree of hemispheric specialisation for a task. 
Running Head: Leftward bias and judging attractiveness

\section{References}

Atalay, A. S., Bodur, H. O., \& Rasolofoarison, D. (2012). Shining in the Center: Central Gaze Cascade Effect on Product Choice. Journal of Consumer Research, 39(4), 848866. doi:10.1086/665984.

Beaumont, J. G. (1985). Lateral organization and aesthetic preference: The importance of peripheral visual asymmetries. Neuropsychologia, 23(1), 103-113.

Berlyne, D. E. (1963). Complexity and incongruity variables as determinants of exploratory choice and evaluative ratings. Canadian Journal of Psychology, 17, 274-290.

Bindemann, M. (2010). Scene and screen centre bias early eye movements in scene viewing. Vision Research, 50, $2577-2587$.

Boles, D. B., \& Karner, T. A. (1996). Hemispheric differences in global versus local processing: Still unclear. Brain and Cognition, 30(2), 232-243.

Boles, D. B., Barth, J. M., \& Merrill, E. C. (2008). Asymmetry and performance: Toward a neurodevelopmental theory. Brain and cognition, 66(2), 124-139.

Bourne, V. J. (2008). Examining the relationship between degree of handedness and degree of cerebral lateralization for processing facial emotion. Neuropsychology, 22(3), 350.

Bourne, V. J. (2010). How are emotions lateralised in the brain? Contrasting existing hypotheses using the chimeric faces test. Cognition and Emotion, 24(5), 903-911.

Bowers, D., \& Heilman, K. M. (1980). Pseudoneglect: effects of hemispace on a tactile line bisection task. Neuropsychologia, 18(4), 491-498.

Bradshaw, J. L., \& Nettleton, N. C. (1981). The nature of hemispheric specialization in man. Behavioral and Brain Sciences, 4(01), 51-63. 
Running Head: Leftward bias and judging attractiveness

Bradshaw, J. L., Nathan, G., Nettleton, N. C., Wilson, L., \& Pierson, J. (1987). Why is there a left side underestimation in rod bisection? Neuropsychologia, 25(4), 735-738.

Brodie, E. E., \& Pettigrew, L. E. (1996). Is left always right? Directional deviations in visual line bisection as a function of hand and initial scanning direction. Neuropsychologia, 34(5), 467-470.

Bryden, M. P. (1982). Laterality: Functional asymmetry in the intact brain. New York, NY: Academic Press.

Bryden, M. P., \& Mondor, T. A. (1991). Attentional factors in visual field asymmetries. Canadian Journal of Psychology, 45, 427-447. doi:10.1037/h0084305

Burt, D. M., \& Perrett, D. I. (1997). Perceptual asymmetries in judgements of facial attractiveness, age, gender, speech and expression. Neuropsychologia, 35, 685-693. doi:10. 1016/S0028-3932(96)00111-X

Calvo, M. G., \& Nummenmaa, L. (2008). Detection of emotional faces: salient physical features guide effective visual search. Journal of Experimental Psychology: General, 137(3), 471.

Chahboun, S., Flumini, A., Pérez González, C., McManus, I. C., \& Santiago, J. (2017). Reading and writing direction effects on the aesthetic appreciation of photographs. Laterality: Asymmetries of Body, Brain and Cognition, 22(3), 313-339. doi: 10.1080/1357650X.2016.1196214

Chiang, C. H., Ballantyne, A. O., \& Trauner, D. A. (2000). Development of perceptual asymmetry for free viewing of chimeric stimuli. Brain and Cognition, 44(3), 415424. 
Running Head: Leftward bias and judging attractiveness

Charles, J., Sahraie, A., \& McGeorge, P. (2007). Hemispatial asymmetries in judgment of stimulus size. Perception \& Psychophysics, 69(5), 687-698.

Chokron, S., Bartolomeo, P., Perenin, M. T., Helft, G., \& Imbert, M. (1998). Scanning direction and line bisection: A study of normal subjects and unilateral neglect patients with opposite reading habits. Cognitive Brain Research, 7(2), 173-178.

Chokron, S., \& De Agostini, M. (2000). Reading habits influence aesthetic preference. Cognitive Brain Research, 10(1), 45-49.

Christman, S. D., \& Hackworth, M. D. (1993). Equivalent perceptual asymmetries for free viewing of positive and negative emotional expressions in chimeric faces. Neuropsychologia, 31, 621-624.

Coolican, J., Eskes, G. A., McMullen, P. A., \& Lecky, E. (2008). Perceptual biases in processing facial identity and emotion. Brain and Cognition, 66(2), 176-187.

Danilova, M. V., \& Mollon, J. D. (2009). The symmetry of visual fields in chromatic discrimination. Brain and Cognition, 69(1), 39-46.

De Agostini, M., Kazandjian, S., Cavezian, C., Lellouch, J., \& Chokron, S. (2010). Visual aesthetic preference: Effects of handedness, sex, and age-related reading/writing directional scanning experience. Writing Systems Research, 2(2), 77-85.

Gazzaniga, M. S. (1998). The split brain revisited. Scientific American, 279(1), 50-55.

Heath, R., Rouhana, A., \& Abi Ghanem, D. (2005). Asymmetric bias in perception of facial affect among Roman and Arabic script readers. Laterality: Asymmetries of Body, Brain, and Cognition, 10(1), 51-64. 
Innes, B., Burt, D. M., Birch, Y. K., \& Hausmann, M. (2015). A leftward bias however you look at it: Revisiting the emotional chimeric face task as a tool for measuring emotion lateralization. Laterality: Asymmetries of Body, Brain and Cognition, 1-19.

Ishii, Y., Okubo, M., Nicholls, M. E., \& Imai, H. (2011). Lateral biases and reading direction: A dissociation between aesthetic preference and line bisection. Brain and Cognition, 75(3), 242-247.

Jewell, G., \& McCourt, M. E. (2000). Pseudoneglect: a review and meta-analysis of performance factors in line bisection tasks. Neuropsychologia, 38(1), 93-110.

Kimura, D. (1961). Cerebral dominance and the perception of verbal stimuli. Canadian Journal of Psychology, 15, 166-171. doi:10.1037/h0083219

Kinsbourne, M. (1970). The cerebral basis of lateral asymmetries in attention. Acta psychologica, 33, 193-201.

Kinsbourne, M. (1993). Orientational bias model of unilateral neglect: evidence from attentional gradients within hemispace. Unilateral neglect: Clinical and experimental studies, 63-86.

Kreplin, U., Thoma, V., \& Rodway, P. (2014). Looking behaviour and preference for artworks: The role of emotional valence and location. Acta psychologica, 152, 100-108.

Leder, H., Belke, B., Oeberst, A., \& Augustin, D. (2004). A model of aesthetic appreciation and aesthetic judgments. British Journal of Psychology, 95(4), 489-508.. doi:10.1348/0007126042369811

Levine, S. C., \& Levy, J. (1986). Perceptual asymmetry for chimeric faces across the life span. Brain and Cognition, 5(3), 291-306.

Levy, J., Heller, W., Banich, M. T., \& Burton, L. A. (1983). Asymmetry of perception in free viewing of chimeric faces. Brain and cognition, 2(4), 404-419. 
Luh, K. E., Rueckert, L. M., \& Levy, J. (1991). Perceptual asymmetries for free viewing of several types of chimeric stimuli. Brain and Cognition, 16(1), 83-103.

Manning, L., Halligan, P. W., \& Marshall, J. C. (1990). Individual variation in line bisection: a study of normal subjects with application to the interpretation of visual neglect. Neuropsychologia, 28(7), 647-655.

Marinsek N., Turner B. O., Gazzaniga M., \& Miller M. B. (2014). Divergent hemispheric reasoning strategies: reducing uncertainty versus resolving inconsistency. Frontiers in Human Neuroscience, 8:839.10.3389/fnhum.2014.0083

Mattingley, J. B., Bradshaw, J. L., Nettleton, N. C., Bradshaw, J. A. (1994). Can task specific perceptual bias be distinguished from unilateral neglect? Neuropsychologia, 32, 805817.

McManus I.C. (1980). The aesthetics of simple figures. Brit. J. Psychol. 71:502-24.

Nebes, R. D. (1978). Direct examination of cognitive function in the right and left hemispheres. Asymmetrical function of the brain, 99-140.

Nicholls, M. E., Bradshaw, J. L., \& Mattingley, J. B. (1999). Free-viewing perceptual asymmetries for the judgement of brightness, numerosity and size. Neuropsychologia, 37(3), 307-314.

Nicholls, M. E., Hobson, A., Petty, J., Churches, O., \& Thomas, N. A. (2017). The effect of cerebral asymmetries and eye scanning on pseudoneglect for a visual search task. Brain and Cognition, 111, 134-143.

Nicholls, M. E., \& Roberts, G. R. (2002). Can free-viewing perceptual asymmetries be explained by scanning, pre-motor or attentional biases? Cortex, 38(2), 113-136.

Oltedal, L., \& Hugdahl, K. (2016). Opposite brain laterality in analogous auditory and visual tests. Laterality: Asymmetries of Body, Brain and Cognition, 1-13. 
Pelowski, M., Markey, P. S., Lauring, J. O., \& Leder, H. (2016). Visualizing the impact of art: An update and comparison of current psychological models of art experience. Frontiers in Human Neuroscience, 10.

Reber, R., Schwarz, N., \& Winkielman, P. (2004). Processing fluency and aesthetic pleasure: Is beauty in the perceiver's processing experience? Personality and Social Psychology Review, 8(4), 364-382.doi:10.1207/s15327957pspr0804_3

Reuter-Lorenz, P. A., Kinsbourne, M., \& Moscovitch, M. (1990). Hemispheric control of spatial attention. Brain and cognition, 12(2), 240-266.

Roberts, M. N. (2007). Complexity and aesthetic preference for diverse visual stimuli. Unpublished Doctoral Dissertation. Universitat de les Illes Balears.

Rodway, P., Schepman, A., \& Lambert, J. (2012). Preferring the One in the Middle: Further Evidence for the Centre-stage Effect. Applied Cognitive Psychology, 26(2), 215-222. doi:10.1002/acp.1812.

Rossion, B., Bodart, J.M., Pourtois, G., Thioux, M., Bol, A., Cosnard, G., et al. (2000). Functional imaging of visual semantic processing in the human brain. Cortex, 36, 579 591.doi:10.1016/s0010-9452(08)70539-2

Sasaki, H., Morimoto, A., Nishio, A., \& Matsuura, S. (2007). Right hemisphere specialization for color detection. Brain and Cognition, 64(3), 282-289.

Schepman, A., Rodway, P., Pullen, S. J., \& Kirkham, J. (2015). Shared liking and association valence for representational art but not abstract art. Journal of vision, 15(5), 11 11.

Spencer, K. M., \& Banich, M. T. (2005). Hemispheric biases and the control of visuospatial attention: an ERP study. BMC neuroscience, 6(1), 51. 
Running Head: Leftward bias and judging attractiveness

Taylor, S., Workman, L., \& Yeomans, H. (2012). Abnormal patterns of cerebral lateralisation as revealed by the universal chimeric faces task in individuals with autistic disorder. Laterality: Asymmetries of Body, Brain and Cognition, 17(4), 428-437.

Vaid, J., \& Singh, M. (1989). Asymmetries in the perception of facial affect: Is there an influence of reading habits?. Neuropsychologia, 27(10), 1277-1287.

Van Kleeck, M. H. (1989). Hemispheric differences in global versus local processing of hierarchical visual stimuli by normal subjects: New data and a meta-analysis of previous studies. Neuropsychologia, 27(9), 1165-1178.

Voyer, D., Voyer, S. D., \& Tramonte, L. (2012). Free-viewing laterality tasks: A multilevel meta-analysis. Neuropsychology, 26(5), 551.

Watling, D., \& Bourne, V. J. (2013). Sex differences in the relationship between children's emotional expression discrimination and their developing hemispheric lateralization. Developmental neuropsychology, 38(7), 496-506.

Workman, L., Chilvers, L., Yeomans, H., \& Taylor, S. (2006). Development of cerebral lateralisation for recognition of emotions in chimeric faces in children aged 5 to 11 . Laterality: Asymmetries of Body, Brain and Cognition, 11(6), 493-507.

Workman, L., Peters, S., \& Taylor, S. (2000). Lateralisation of perceptual processing of pro-and anti-social emotions displayed in chimeric faces. Laterality: Asymmetries of Body, Brain and Cognition, 5(3), 237-249.

Worley, M. M., \& Boles, D. B. (2016). The face is the thing: Faces, not emotions, are responsible for chimeric perceptual asymmetry. Laterality: Asymmetries of Body, Brain and Cognition, 1-17. 
Running Head: Leftward bias and judging attractiveness

Wright, D., Makin, A. D., \& Bertamini, M. (2017). Electrophysiological responses to symmetry presented in the left or in the right visual hemifield. Cortex, 86, 93-108.

Zaidel, D. W., \& Kasher, A. (1989). Hemispheric memory for surrealistic versus realistic paintings. Cortex, 25(4), 617-641.

Zaidel, D. W. \& Edelstyn, N. (1995). Hemispheric Semantics: Effects of Pictorial Organization on Patients with Unilateral Brain Damage. International Journal of Neuroscience, 82(3-4), 215-221.

Zaidel, D. W. (2013).Split-brain, the right hemisphere and art: fact and fiction. Prog.Brain Res. 204, 3-17.doi:10.1016/b978-0-444-63287-6.00001-4

Zaidel, D. W. (2015). Hemispheric specialization, art, and aesthetics. In, Art, Aesthetics, and the Brain, M. Nadal, J. P. Huston, L. Agnati, F. Mora, C. J. Cela-Conde (Eds.), Oxford: Oxford University Press. 

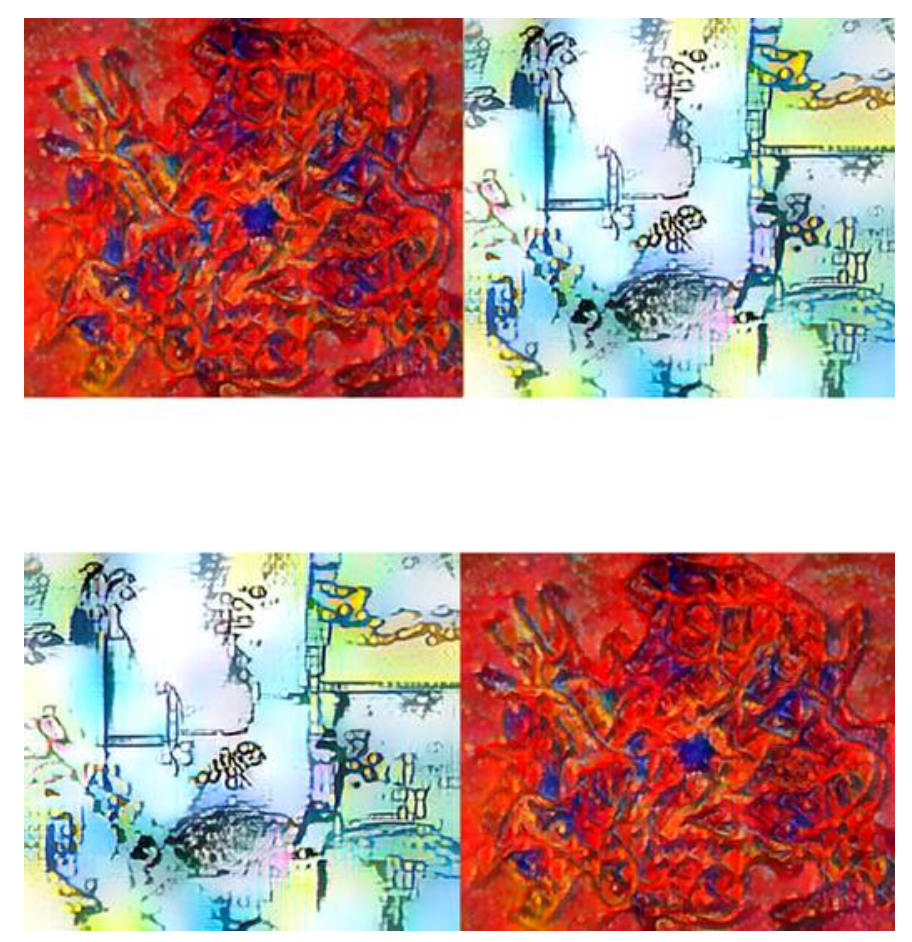

Figure 1.

Example of how the chimeric artworks were displayed. This example is not of actual stimuli used in the experiment, which we are unable to show for copyright reasons. The stimuli are available in the supplementary materials. 
Running Head: Leftward bias and judging attractiveness

Table 1. Laterality quotient, with standard error, for each age group.

\begin{tabular}{ccc}
\hline Age & Laterality Quotient & Standard Error \\
\hline 5 to 6 & -.0317 & .046 \\
\hline 9 to 10 & .0412 & .056 \\
\hline 10 to 11 & .124 & .050 \\
\hline
\end{tabular}

Table 2. Mean ratings for each category of wallpaper design. Standard errors in brackets.

Design

\begin{tabular}{ccccc}
\hline & Attractive & Unattractive & Attractive & Unattractive \\
& Colour & Colour & Non-colour & Non-Colour \\
\hline Complexity & $4.5(.20)$ & $4.3(.20)$ & $5.0(.21)$ & $4.3(.26)$ \\
\hline Luminance & $3.8(.16)$ & $3.8(.19)$ & $2.5(.19)$ & $2.3(.13)$ \\
\hline Colourfulness & $3.6(.14)$ & $3.3(.22)$ & $1.5(.12)$ & $1.3(.11)$
\end{tabular}


Running Head: Leftward bias and judging attractiveness

Table 3. Mean ratings, with standard errors, for the three attractive and three unattractive designs.

\section{Design}

Attractive $\quad$ Standard Error $\quad$ Unattractive $\quad$ Standard Error

\begin{tabular}{lcccc}
\hline Attractiveness & $4.4,4.3,4.4$ & $.8, .63, .43$ & $1.6,2.5,1.4$ & $.17, .5, .24$ \\
& & & & \\
Complexity & $3.9,4.4,3.6$ & $.37, .32, .34$ & $4.9,5.4,3.6$ & $.49, .34, .25$ \\
& & & & \\
\hline Colourfulness & $3.5,5.3,2.4$ & $.32, .24, .25$ & $4.7,3.0,2.9$ & $.21, .25, .31$ \\
& & & & \\
\hline Luminance & $3.1,5.1,2.9$ & $.36, .28, .27$ & $5.8,3.4,3.7$ & $.22, .31, .39$
\end{tabular}

